\title{
Management of fistula between neobladder and rectum using an atrial septal defect closure device
}

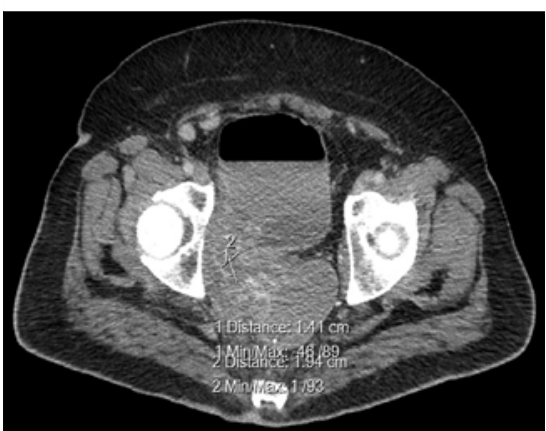

- Fig. 1 Contrast-enhanced computed tomography of the abdomen showing a fistulous opening measuring $1.4 \mathrm{~cm} \times$ $1.9 \mathrm{~cm}$ between neobladder and rectum.

Radiation therapy is widely used to treat malignancies in the pelvic cavity such as cancers of the colorectum, urinary tract, uterus, adnexa, and prostate. Studies have reported pelvic radiotherapy to have been closely associated with gastrointestinal complications such as mucosal ulcers, stenosis, perforation, and fistula formation [1].

These fistulous tracts are life-threatening since the patient is in a perpetual state of urosepsis. Surgical interventions are risky due to underlying advanced disease. Therefore, the management of an enterovesical fistula between a surgical neobladder and the rectum in patients previously operated on for bladder malignancy is challenging [2]. We present the case of a 58-year-old man who underwent surgery for bladder carcinoma with construction of a neobladder. The surgery was followed by chemoradiation therapy.

The patient presented in the emergency department with a 15-day history of fever, hypotension, pneumaturia, and fecaluria. Contrast-enhanced computed tomography of the abdomen revealed a fistulous opening measuring $1.4 \mathrm{~cm} \times$ $1.9 \mathrm{~cm}$ between neobladder and rectum ( Fig.1). Successful endoscopic closure
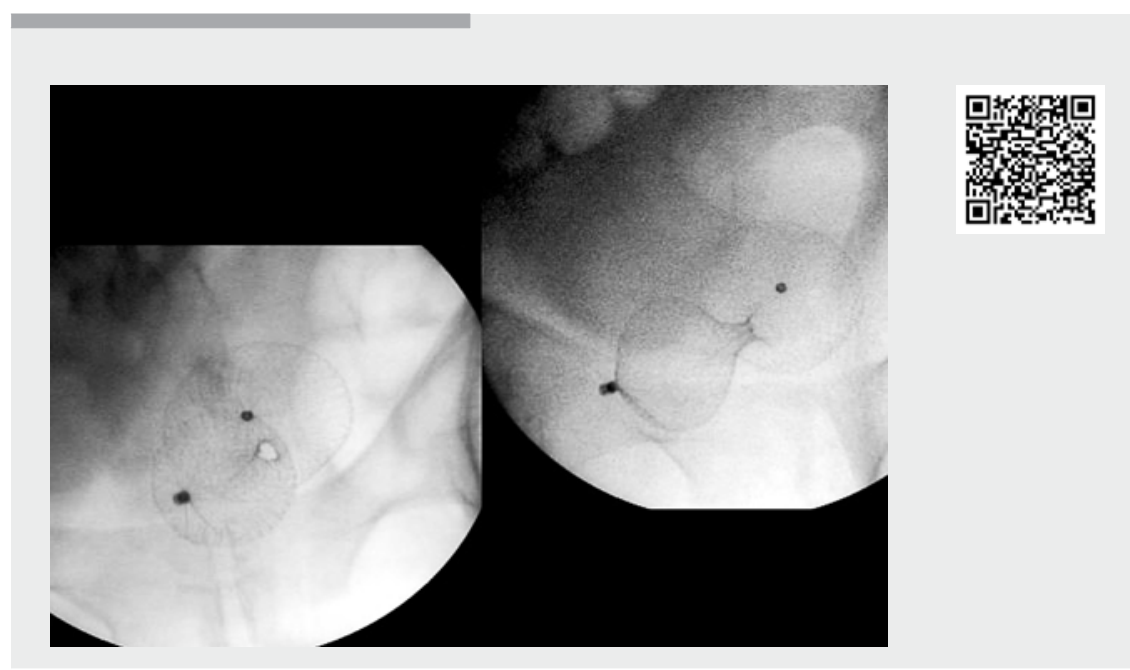

Video 1 Management of fistula between neobladder and rectum using an atrial septal defect closure device.

of the rectovesical fistula was achieved using an atrial septal defect closure device ( $\downarrow$ Video 1 ). Following the procedure, the urosepsis and hypotension improved and the patient was catheterized for 3 days. The patient had clear urine on the 3 rd day and a culture after 72 hours was sterile. He was discharged on the 7 th day. He remained well for one and a half months before succumbing to his underlying advanced malignancy.

$$
\text { Endoscopy_UCTN_Code_TTT_1AQ_2A] }
$$

\section{Competing interests}

The authors declare that they have no conflict of interest.

The authors

\section{Ashlee Sidhu, Nagoor Basha Shaik, Malay Sharma}

Aryavart Hospital, Daurala Sugar Mills, Meerut, Uttar Pradesh, India
Corresponding author

Ashlee Sidhu, MD

Aryavart Hospital, NH334, Daurala Sugar Mills, Meerut, Uttar Pradesh - 250110, India ashlee2907@gmail.com

\section{References}

[1] Iwamuro M, Hasegawa K, Hanayama Y et al. Enterovaginal and colovesical fistulas as late complications of pelvic radiotherapy. J Gen Fam Med 2018; 19: 166-169

[2] Golabek T, Szymanska A, Szopinski T et al. Enterovesical fistulae: aetiology, imaging, and management. Gastroenterol Res Pract 2013; 2013: 617967

Bibliography

Endoscopy 2021; 53: 1090

DOI 10.1055/a-1298-3906

ISSN 0013-726X

published online 26.11 .2020

(c) 2021. Thieme. All rights reserved.

Georg Thieme Verlag KG, Rüdigerstraße 14,

70469 Stuttgart, Germany 